H. E. HESS - E. LANDOLT - R. HIRZEL - FLORA DER SCHWEIZ - BAND 2 


\section{Flora der Schweiz}

Dr. Hans Ernst Heß

Professor für spezielle Botanik

an der Eidgenössischen Technischen Hochschule

in Zürich

\section{Dr. Elias Landolt}

Professor für Geobotanik

an der Eidgenössischen Technischen Hochschule in Zürich

Rosmarie Hirzel

Zürich

(Zeichnungen)

Springer Basel AG

\section{und angrenzender Gebiete}

Band 2: Nymphaeaceae bis Primulaceae

Zweite, durchgesehene Auflage 


\section{GIP-Kurztitelaufnahme der Deutschen Bibliothek}

\section{Heß, Hans Ernst}

Flora der Schweiz und angrenzender Gebiete

Hans Ernst $\mathrm{He} B$; Elias Landolt. Rosmarie

Hirzel (Zeichn.).

NE: Landolt, Elias:

Bd. 2. Nymphaeaceae bis Primulaceae. -

2., durchges. Aufl. - 1977.

Fotolithos: Walter Nievergelt, Zürich

Buchgestaltung: Albert Gomm

Gesamtherstellung: Birkhäuser AG, Basel

(C) Springer Basel AG 1970, 1977

Originally published by Birkhäuser Verlag Basel in 1977

ISBN 978-3-0348-5107-7 ISBN 978-3-0348-5105-3 (eBook)

DOI 10.1007/978-3-0348-5105-3 
Inhaltsübersicht zu Band 2

Familien

7 Nymphaeaceae

11 Ceratophyllaceae

13 Ranunculaceae

103 Berberidaceae

105 Magnoliaceae

107 Lauraceae

107 Papaveraceae

117 Fumariaceae

124 Capparaceae

124 Cruciferae

249 Sarraceniacea

249 Droseraceae

253 Crassulaceae

271 Saxifragaceae

307 Platanaceae

309 Rosaceae

480 Leguninosae

615 Geraniaceae

632 Oxalidacea

634 Linaceae

638 Zygophyllaceae

639 Tropaeolaceae

640 Rutaceae

643 Simaroubaceae

643 Polygalaceae

650 Euphorbiaceae

670 Callitrichaceae

674 Buxaceae

675 Anacardiaceae

678 Aquifoliaceae

679 Celastraceae
682 Staphyleaceae

83 Aceraceae

688 Hippocastanaceae

689 Balsaminaceae

693 Rhamnaceae

697 Vitaceae

700 Tiliaceae

702 Malvaceae

709 Hypericaceae

718 Elatinaceae

720 Tamaricaceae

721 Cistaceae

729 Violaceae

750 Cactaceae

752 Thymelaeaceae

755 Elaeagnacea

756 Lythraceae

760 Punicaceae

761 Myrtaceae

762 Oenotheraceae

782 Trapaceae

783 Haloragaceae

785 Hippuridaceae

786 Araliaceae

787 Umbelliferae

890 Cornaceae

892 Pyrolaceae

898 Empetraceae

900 Ericaceae

913 Primulaceae

945 Lateinische Namen

955 Deutsche Namen 